\title{
The Influence of Psychosocial Constructs on the Adherence to Active Surveillance for Localized Prostate Cancer in a Prospective, Population-based Cohort
}

\author{
Maximilian F. Lang, Mark D. Tyson, JoAnn Rudd Alvarez, Tatsuki Koyama, Karen E. \\ Hoffman, Matthew J. Resnick, Matthew R. Cooperberg, Xiao-Cheng Wu, Vivien Chen, Lisa \\ E. Paddock, Ann S. Hamilton, Mia Hashibe, Michael Goodman, Sheldon Greenfield, Sherrie \\ H. Kaplan, Antoinette Stroup, David F. Penson, and Daniel A. Barocas \\ Vanderbilt University School of Medicine, Nashville, TN; the Department of Urologic Surgery, \\ Vanderbilt University Medical Center, Nashville, TN; the Department of Biostatistics, Vanderbilt \\ University School of Medicine, Nashville, TN; the Department of Radiation Oncology, The \\ University of Texas MD Anderson Cancer Center, Houston, TX; the Department of Health Policy, \\ Vanderbilt University School of Medicine, Nashville, TN; the The Geriatric Research, Education, \\ and Clinical Center, Tennessee Valley Veterans Affairs Health Care System, Nashville, TN; the \\ Department of Urology, Helen Diller Family Comprehensive Cancer Center, University of \\ California, San Francisco, CA; the Epidemiology and Biostatistics, Helen Diller Family \\ Comprehensive Cancer Center, University of California, San Francisco, CA; the School of Public \\ Health, Louisiana State University Health Sciences Center, New Orleans, LA; the Rutgers Cancer \\ Institute of New Jersey, Cancer Surveillance Research Program, New Brunswick, NJ; the \\ Department of Preventive Medicine, Keck School of Medicine, University of Southern California, \\ Los Angeles, CA; the Department of Family and Preventive Medicine and Huntsman Cancer \\ Institute, University of Utah, Salt Lake City, UT; the Department of Epidemiology, Rollins School of \\ Public Health, Emory University, Atlanta, GA; the Center for Health Policy Research and \\ Department of Medicine, University of California Irvine, Irvine, CA; the Health Policy Research \\ Institute, University of California Irvine, Irvine, CA; and the Rutgers Cancer Institute of New \\ Jersey, Rutgers University, New Brunswick, NJ
}

\section{Abstract}

OBJECTIVE-To evaluate the influence of psychosocial factors such as prostate cancer (PCa) anxiety, social support, participation in medical decision-making (PDM), and educational level on patient decisions to discontinue PCa active surveillance (AS) in the absence of disease progression.

METHODS-The Comparative Effectiveness Analysis of Surgery and Radiation study is a prospective, population-based cohort study of men with localized PCa diagnosed in 2011-2012. PCa anxiety, social support, PDM, educational level, and patient reasons for discontinuing AS

Address correspondence to: Maximilian F. Lang, BS, Vanderbilt University School of Medicine, 2215 Garland Ave (Light Hall), Nashville, TN 37232. maximilian.f.lang@ vanderbilt.edu.

Financial Disclosure: The authors declare that they have no relevant financial interests. 
were assessed through patient surveys. A Cox proportional hazards model examined the relationship between psychosocial variables and time to discontinuation of AS.

RESULTS-Of 531 patients on AS, 165 (30.9\%) underwent treatment after median follow-up of 37 months. Whereas $69 \%$ of patients cited only medical reasons for discontinuing AS, $31 \%$ cited at least 1 personal reason, and $8 \%$ cited personal reasons only. Patients with some college education discontinued AS significantly earlier (hazard ratio: 2.0, 95\% confidence interval: 1.2, 3.2) than patients with less education. PCa anxiety, social support, and PDM were not associated with seeking treatment.

CONCLUSION-We found that $31 \%$ of men who choose AS for PCa discontinue AS within 3 years. Eight percent of men who sought treatment did so in the absence of disease progression. Education, but not psychosocial factors, seems to influence definitive treatment-seeking. Future research is needed to understand how factors unrelated to disease severity influence treatment decisions among patients on AS to identify opportunities to improve adherence to AS.

Active surveillance (AS) has become a preferred treatment option for men with low-risk prostate cancer (PCa). ${ }^{1,2}$ However, up to one third of men will discontinue AS in favor of definitive treatment within 5 years. ${ }^{3,4}$ Although most men who later choose treatment do so in the context of clinical progression, ${ }^{5}$ psychosocial factors may nonetheless drive patients under AS toward definitive treatment. ${ }^{6,7}$ Whereas a decision to discontinue AS may be driven by disease progression, ${ }^{8}$ the impact of psychosocial constructs such as anxiety, social support, and participation in medical decision-making (PDM) on the course of AS has received little attention in literature to date.

Recent studies suggest that of the men who choose AS for PCa management, approximately 20\%-33\% will discontinue AS in favor of definitive treatment, including some who are without signs of disease progression. $., 9,10$ At present, no population-based study has described the psychosocial characteristics of patients who are likely to discontinue AS. Because PCa treatments carry a risk of side effects, ${ }^{11}$ it is important that providers understand the effect of psychosocial factors on adherence to AS.

In this context, this population-based analysis sought to understand why patients decide to discontinue AS and identify patient characteristics that may influence these decisions. We hypothesized that lower social support and PDM scores and higher PCa anxiety scores would be associated with the discontinuation of AS. We also hypothesized that educational level, as a surrogate for understanding the risk of disease progression without treatment relative to the risk of treatment side effects, may influence discontinuation of AS. If so, one could identify patients with psychosocial or demographic determinants of AS discontinuation and target them for interventions designed to improve adherence, thereby reducing the likelihood of discontinuing AS in the absence of clinical progression.

\section{METHODS}

\section{Population}

The Comparative Effectiveness Analysis of Surgery and Radiation (CEASAR) cohort is an ongoing prospective, population-based, observational study assessing the comparative 
benefits and harms of management strategies for localized PCa. The CEASAR accrued patients from 2011 to 2012 and included 3269 patients from 5 Surveillance, Epidemiology, and End Results catchment areas (Louisiana, New Jersey, Atlanta, Utah, and Los Angeles). ${ }^{12}$ An additional 238 patients from the Cancer of the Prostate Strategic Urologic Research Endeavor (CaPSURE) database, a prospective PCa registry, were included to examine men receiving novel treatment strategies. CaPSURE includes men from 43 community and academic urology practices in the United States. ${ }^{13}$ Inclusion criteria were men aged 80 years or younger diagnosed with PCa within 6 months of enrollment, prostatespecific antigen (PSA) less than $50 \mathrm{ng} / \mathrm{dL}$, and clinical stage cT1 or cT2. For this analysis, we excluded patients with D'Amico high-risk disease because they are not appropriate candidates for AS.

\section{AS Cohort and Survey Instruments}

The AS cohort included men with low or intermediate D'Amico risk disease who had no treatment within 12 months of diagnosis. CEASAR study patients completed an enrollment survey as well as 6-month, 12-month, and 36-month surveys. Men who had no treatment within 6 months of diagnosis and either chose AS on a survey or had chart documentation of choosing AS were also included. Information from the 12- and 36-month surveys, Surveillance, Epidemiology, and End Results registries, and medical charts was used to determine whether AS patients subsequently underwent definitive treatment. Patients who indicated they underwent treatment on the 12-month or 36-month survey or had record of treatment in the medical chart or registry were considered to have discontinued AS at the date of their treatment, and patients who did not report treatment were considered to be continuing with AS.

Men were assessed at baseline on social support, PCa-related anxiety, and PDM levels among other demographic, psychosocial, general health, and disease-related factors that could influence treatment choice or treatment outcome. Social support was assessed using questions from the Medical Outcomes Study Social Support Survey, which reflect patients' perceptions of their social support networks, with higher scores indicating more social support. ${ }^{14} \mathrm{PCa}$ anxiety was assessed using questions from scales developed by Clark et al, which measure patient perceptions of their PCa treatment, ${ }^{15}$ yielding a $0-100$ score, with higher scores indicating more concern over disease control. Patients' participation in treatment decisions was assessed using the PDM scale, a survey that asks patients to rate how their physicians involve them in decisions and encourage patient responsibility in care, with higher scores indicating higher levels of PDM. ${ }^{16}$ Disease characteristics and treatments were obtained from a chart review done at the time of the 12-month survey. The 3-year CEASAR survey asked patients their reasons for discontinuing AS in favor of definitive treatment. Patients self-reported their educational level, race, and marital status. Validated survey instruments were used to assess patient comorbidities (Total Illness Burden Index to Prostate Cancer [TIBI-CaP $]^{17}$ ) and general- and disease-specific health-related quality of life (Expanded Prostate Cancer Index Composite ${ }^{18}$ ). 


\section{Statistical Analysis}

Patients in the AS cohort were compared with patients who initially chose definitive treatment on several patient and disease characteristics and on pretreatment function using summary statistics. Patient-reported reasons for seeking definitive treatment were tabulated. The distribution of times to discontinuing AS was characterized with a Kaplan-Meier survival curve with confidence intervals calculated using Greenwood's standard error estimator. To identify predictors of discontinuing AS, we fit a Cox proportional hazard model with time to definitive treatment as the response with the following covariates selected a priori: social support, PCa anxiety, PDM, age, TIBI comorbidities, education, race (black vs nonblack), and marital status. The results of Cox models were expressed as adjusted hazard ratios (HR) and the corresponding 95\% confidence intervals (CIs). Analyses were conducted using $\mathrm{R}$ version 3.2.2. ${ }^{19}$

\section{RESULTS}

\section{Study Cohort}

Overall, 3269 patients met inclusion criteria for the CEASAR study, 590 (17.9\%) of whom met criteria for AS. After excluding high-risk patients, 534 patients remained, of whom 419 (79\%) had low-risk disease and $112(21 \%)$ had intermediate-risk disease. Within the AS cohort, $386(72 \%)$ patients returned a 3-year survey, and median follow-up time was 37.2 (Q1, Q3: 17.1, 38.5) months.

Comparisons between patients who chose AS and those who chose other treatments are shown in Table S1. Patients on AS were older, less likely to have private insurance, less likely to work full time, and had more education. The 3-year survey participation rate was lower in AS patients than in the treatment cohort (72\% vs 78\%). Expanded Prostate Cancer Index Composite domain scores of the pretreatment sexual, urinary, and bowel function of the AS cohort in comparison with the definitive treatment cohort are found in Table S2.

\section{Discontinuation of Active Surveillance}

There were 165 (30.9\%) patients in the AS cohort who discontinued AS in favor of definitive treatment within the follow-up period. As shown in the Kaplan-Meier curve (Fig. 1), the probabilities of patients remaining on AS at 1,2, and 3 years were $89 \%$ (95\% CI: 86\%, 92\%), 72\% (95\% CI: 68\%, 77\%), and 66\% (95\% CI: 62\%, 71\%), respectively. Most patients who discontinued AS chose surgery (77 [47\%]) or radiation (70 [42\%]). Of 165 patients who discontinued AS, 141 (85\%) returned 3-year surveys. On this survey, 77 (55\%) patients indicated specific reasons for why they discontinued AS. Within that group, 53 (69\%) patients cited medical reasons alone, 6 (8\%) cited personal reasons alone, and 18 (23\%) cited both a medical and a personal reason for discontinuing AS. Table 1 shows a breakdown of reasons patients gave.

\section{Prognostic Factors for Discontinuing AS}

On multivariable analysis, patients with some college education discontinued AS significantly earlier than patients who had high school or less education ( $\mathrm{HR}=2.0,95 \% \mathrm{CI}$ : 1.2-3.2). The other 3 psychosocial factors (PCa anxiety, social support, and PDM), race, 
marital status, age, and TIBI score did not have a clear influence on discontinuation of AS. HRs estimated from our proportional hazards model are shown in Figure 2.

\section{DISCUSSION}

In this population-based observational study, we found that men who selected AS for PCa management had a 33\% chance of receiving definitive treatment within 3 years, and that $8 \%$ of men who discontinued AS reported only personal reasons for pursuing definitive treatment. We did not identify statistically significant associations between psychosocial constructs such as social support, PCa anxiety, and PDM and discontinuing AS.

Identifying risk factors for discontinuation of AS in the absence of disease progression could facilitate the development of tools to help vulnerable men adhere to AS. Such interventions would be beneficial because men who remain on AS have stable disease-specific function and quality of life, whereas those who are treated are at significant risk for deterioration of urinary, bowel, and erectile function. ${ }^{11,20}$ However, the relationship between psychosocial factors and discontinuation of AS remains uncertain.

In some respects, our study corroborates previous findings. However, $33 \%$ of patients in the CEASAR cohort discontinued AS in favor of definitive treatment. This result puts our study at the higher range of previous studies that have demonstrated that about $20 \%-33 \%$ of men will discontinue AS within 3-5 years. $3,8,9,21$ We also found that $31 \%$ of patients in the CEASAR cohort who discontinued AS had one or more personal reasons for doing so. Past studies of AS adherence have also shown that most patients who discontinue AS do so in the context of disease progression, with a smaller proportion discontinuing out of personal preference. ${ }^{21,22}$ For example, in the Prostate Cancer Research International Active Surveillance study, $26.6 \%$ of patients who discontinued AS did so for reasons other than disease progression, including $9 \%$ who discontinued AS because of anxiety. ${ }^{21}$ The Swedish population-based study of the National Prostate Cancer Registry found that the proportion of men who discontinued AS for personal preference was 20\% (108 of 530 with documented reason for discontinuation). ${ }^{8}$ Additionally, this study identified high educational levels as one of the predictors of discontinuing AS for personal preference, which is corroborated by our finding that higher educational level is associated with discontinuing AS earlier.

However, the Swedish study did not contain data on anxiety or other psychosocial measures. Studies from academic centers in the United States have found patients with PCa discontinued AS in the absence of disease progression in $9 \%-23 \%$ of cases. ${ }^{3,22-24}$ To our knowledge, our analysis remains the only population-based analysis of American patients to date and one of the only analyses that includes patient-reported PCa anxiety, social support, and PDM.

Few studies have fully investigated the influence of psychosocial factors on adherence to AS. The Prostate Cancer Research International Active Surveillance study quantified anxiety, depression, and decisional conflict among 150 men on AS and determined that although $14 \%$ (6 of 42) of men who discontinued did so because of anxiety and distress, anxiety scores were low and stable over time and baseline scores did not predict discontinuation of AS. ${ }^{20}$ Another study found that "neurotic personality scores" correlated 
with anxiety levels while on AS, but anxiety levels did not correlate with discontinuation of AS. ${ }^{6}$ Only 1 previous study within the CaPSURE database found that a psychosocial factor, "anxiety change rate," predicted discontinuation, but did not assess psychosocial variables other than anxiety at baseline. ${ }^{7}$ Our study offers several novel insights into the relationship between patient-level psychosocial characteristics and risk of discontinuation of AS. First, this study is population-based and is more broadly generalizable. Second, we gathered detailed information regarding why patients discontinued AS, which facilitated a descriptive analysis of the most common reasons patients report for undergoing treatment after starting on AS. Lastly, the CEASAR study used patient-reported data to prospectively measure psychosocial variables with validated instruments. This enabled us to test the hypothesis that PCa anxiety, social support, and PDM along with demographic factors contribute to the risk of discontinuing AS. Our hypothesis was not confirmed by the data, which could reflect a true absence of such an association or limited statistical power to detect a difference.

We took a novel approach in attempting to identify factors that influence adherence to AS, but our results should be viewed in the context of methodological limitations within our study's design. Our study, like others examining reasons for discontinuing AS, lacks clinical data that may have influenced treatment decisions (eg number of cores positive and results of post-diagnostic PSAs, biopsies, prostate magnetic resonance imaging, and molecular testing). Additionally, of this cohort, $28 \%$ did not return a 3-year survey, and 25\% of the cohort had less than 1.4 years of follow-up compared with a median of 3.1 years. Of patients who discontinued and completed 3-year surveys, only 77 (55\%) supplied reasons for discontinuing AS. These factors limit interpretation of the influence of psychosocial factors such as education on discontinuing AS in the absence of disease progression. However, similar patterns of follow-up and discontinuation of AS within 3 years are found in previous studies on this topic, and the 77 patients supplying reasons for discontinuation were similar to the 72 nonresponders in all measured characteristics (data not shown). ${ }^{8,23}$ Finally, the impact of several psychosocial elements on discontinuing AS remains unclear after our analyses due to lack of statistical power driven by small sample size. A contributing factor could be heterogeneity in effect directions between people who discontinued AS for different reasons. For example, increased educational attainment could be associated with longer time to discontinuation for personal reasons while not affecting time to discontinuation for progression.

Despite these limitations, our findings may have important clinical implications. Studies aimed at identifying reasons for discontinuing AS could yield results with far-reaching public health impact. Approximately $25 \%$ of patients diagnosed with localized PCa have low-risk disease at the time of diagnosis. ${ }^{25}$ The uptake of AS among men with low-risk disease is increasing, ${ }^{2,26}$ which most observers consider an appropriate response to concerns about overtreatment of low-risk disease. As many as half of eligible men select AS for initial management. ${ }^{27}$ Yet $20 \%-33 \%$ of men discontinue AS within 3-5 years, $8 \%-23 \%$ of whom discontinue for personal preference. . $^{3,8,20-24}$ This is likely a conservative estimate of the influence of personal preference, which may also play a role in men with "soft signs" of progression, such as mild PSA elevation. ${ }^{28}$ Indeed, our study demonstrates this possibility, as 24 of 77 (31\%) patients who supplied a reason for discontinuing AS cited at least 1 personal reason. Thus, a large number of patients may discontinue AS prematurely, and 
improving adherence to AS in appropriate clinical situations could help a substantial number of men. One step toward improved adherence is to identify men at risk for discontinuing AS in the absence of clinical progression. Once these patients are identified, possible interventions to prevent premature discontinuation of AS include PCa education, ${ }^{29}$ anxiety counseling, or outreach and support by medical staff to promote adherence. ${ }^{5}$ Additionally, peer-support groups could be a way to enhance social support and encourage adherence. ${ }^{30}$

\section{CONCLUSION}

This study, using one of the largest population-based cohorts of PCa patients on AS in the United States that is currently available, confirms previous literature that suggested one-third of men will discontinue AS within 3 years, with approximately $10 \%$ discontinuing with no signs of disease progression. Our findings do not definitively answer the question of whether efforts to address PCa anxiety, social support, or PDM could influence adherence to AS, and the relationship of education level to AS adherence remains unclear. Therefore, further studies are warranted to identify the elements of personal preferences, attitudes, demographics, and psychosocial factors that can be leveraged to improve adherence to AS.

\section{Supplementary Material}

Refer to Web version on PubMed Central for supplementary material.

\section{Acknowledgments}

Funding Support: This work was supported by NIH/NCI Grant 1R03CA173812 (DAB). It was also supported by NIH/NCI Grant 5T32CA106183 (MDT); American Cancer Society MSRG-15-103-01-CPHPS (MJR); the US Agency for Healthcare Research and Quality (grants 1R01HS019356 and 1R01HS022640-01); the National Cancer Institute, National Institutes of Health (grant R01-CA114524), and the following contracts to each of the participating institutions: N01-PC-67007, N01-PC-67009, N01-PC-67010, N01-PC-67006, N01-PC-67005, and N01-PC-67000, and through a contract from the Patient-Centered Outcomes Research Institute.

\section{References}

1. Albertsen PC, Hanley JA, Fine J. 20-year outcomes following conservative management of clinically localized prostate cancer. JAMA. 2005; 293:2095-2101. [PubMed: 15870412]

2. Ritch CR, Graves AJ, Keegan KA, et al. Increasing use of observation among men at low risk for prostate cancer mortality. J Urol. 2015; 193:801-806. [PubMed: 25196658]

3. Eggener SE, Mueller A, Berglund RK, et al. A multi-institutional evaluation of active surveillance for low risk prostate cancer. J Urol. 2013; 189:S19-S25. discussion S25. [PubMed: 23234624]

4. Newcomb LF, Thompson IM, Boyer HD, et al. Outcomes of active surveillance for clinically localized prostate cancer in the prospective, multi-institutional Canary PASS cohort. J Urol. 2015; 195:313-320. [PubMed: 26327354]

5. Dall'Era MA, Konety BR, Cowan JE, et al. Active surveillance for the management of prostate cancer in a contemporary cohort. Cancer. 2008; 112:2664-2670. [PubMed: 18433013]

6. van den Bergh RCN, Essink-Bot M-L, Roobol MJ, et al. Anxiety and distress during active surveillance for early prostate cancer. Cancer. 2009; 115:3868-3878. [PubMed: 19637245]

7. Latini DM, Hart SL, Knight SJ, et al. The relationship between anxiety and time to treatment for patients with prostate cancer on surveillance. J Urol. 2007; 178:826-831. discussion 831-2. [PubMed: 17632144]

8. Loeb S, Folkvaljon Y, Makarov DV, et al. Five-year nationwide follow-up study of active surveillance for prostate cancer. Eur Urol. 2015; 67:233-238. [PubMed: 24993868] 
9. Dall'Era MA, Albertsen PC, Bangma C, et al. Active surveillance for prostate cancer: a systematic review of the literature. Eur Urol. 2012; 62:976-983. [PubMed: 22698574]

10. Dall'Era MA, Cooperberg MR, Chan JM, et al. Active surveillance for early-stage prostate cancer: review of the current literature. Cancer. 2008; 112:1650-1659. [PubMed: 18306379]

11. Resnick MJ, Koyama T, Fan K-H, et al. Long-term functional outcomes after treatment for localized prostate cancer. N Engl J Med. 2013; 368:436-445. [PubMed: 23363497]

12. Barocas DA, Chen V, Cooperberg M, et al. Using a population-based observational cohort study to address difficult comparative effectiveness research questions: the CEASAR study. J Comp Eff Res. 2013; 2:445-460. [PubMed: 24236685]

13. Lubeck DP, Litwin MS, Henning JM, et al. The CaPSURE database: a methodology for clinical practice and research in prostate cancer. Urology. 1996; 48:773-777. [PubMed: 8911524]

14. Sherbourne CD, Stewart AL. The MOS social support survey. Soc Sci Med. 1991; 32:705-714. [PubMed: 2035047]

15. Clark JA, Bokhour BG, Inui TS, et al. Measuring patients' perceptions of the outcomes of treatment for early prostate cancer. Med Care. 2003; 41:923-936. [PubMed: 12886172]

16. Kaplan SH, Gandek B, Greenfield S, et al. Patient and visit characteristics related to physicians' participatory decision-making style. Results from the Medical Outcomes Study. Med Care. 1995; 33:1176-1187. [PubMed: 7500658]

17. Stier DM, Greenfield S, Lubeck DP, et al. Quantifying comorbidity in a disease-specific cohort: adaptation of the total illness burden index to prostate cancer. Urology. 1999; 54:424-429. [PubMed: 10475347]

18. Chang P, Szymanski KM, Dunn RL, et al. Expanded prostate cancer index composite for clinical practice: development and validation of a practical health related quality of life instrument for use in the routine clinical care of patients with prostate cancer. J Urol. 2011; 186:865-872. [PubMed: 21788038]

19. R Core Team. R: A Language and Environment for Statistical Computing. Vienna, Austria: R, Foundation for Statistical Computing; 2016. http://www.R-project.org/. U and, No Title

20. Venderbos LDF, van den Bergh RCN, Roobol MJ, et al. A longitudinal study on the impact of active surveillance for prostate cancer on anxiety and distress levels. Psychooncology. 2015; 24:348-354. [PubMed: 25138075]

21. Bul M, Zhu X, Valdagni R, et al. Active surveillance for low-risk prostate cancer worldwide: the PRIAS study. Eur Urol. 2013; 63:597-603. [PubMed: 23159452]

22. Patel MI, DeConcini DT, Lopez-Corona E, et al. An analysis of men with clinically localized prostate cancer who deferred definitive therapy. J Urol. 2004; 171:1520-1524. [PubMed: 15017211]

23. Klotz L, Zhang L, Lam A, et al. Clinical results of long-term follow-up of a large, active surveillance cohort with localized prostate cancer. J Clin Oncol. 2010; 28:126-131. [PubMed: 19917860]

24. Berger ZD, Yeh JC, Carter HB, et al. Characteristics and experiences of patients with localized prostate cancer who left an active surveillance program. Patient. 2014; 7:427-436. [PubMed: 24920082]

25. Barocas DA, Mallin K, Graves AJ, et al. Effect of the USPSTF grade D recommendation against screening for prostate cancer on incident prostate cancer diagnoses in the United States. J Urol. 2015; 194:1587-1593. [PubMed: 26087383]

26. Cooperberg MR, Carroll PR, Klotz L. Active surveillance for prostate cancer: progress and promise. J Clin Oncol. 2011; 29:3669-3676. [PubMed: 21825257]

27. Womble PR, Montie JE, Ye Z, et al. Contemporary use of initial active surveillance among men in Michigan with low-risk prostate cancer. Eur Urol. 2015; 67:44-50. [PubMed: 25159890]

28. Ross AE, Loeb S, Landis P, et al. Prostate-specific antigen kinetics during follow-up are an unreliable trigger for intervention in a prostate cancer surveillance program. J Clin Oncol. 2010; 28:2810-2816. [PubMed: 20439642]

29. Vasarainen H, Lokman U, Ruutu M, et al. Prostate cancer active surveillance and health-related quality of life: results of the Finnish arm of the prospective trial. BJU Int. 2012; 109:1614-1619. [PubMed: 22044485] 
30. Kronenwetter C, Weidner G, Pettengill E, et al. A qualitative analysis of interviews of men with early stage prostate cancer: the Prostate Cancer Lifestyle Trial. Cancer Nurs. 2005; 28:99-107. [PubMed: 15815179]

\section{APPENDIX. SUPPLEMENTARY DATA}

Supplementary data associated with this article can be found, in the online version, at http:// dx.doi.org/10.1016/j.urology.2016.12.063. 


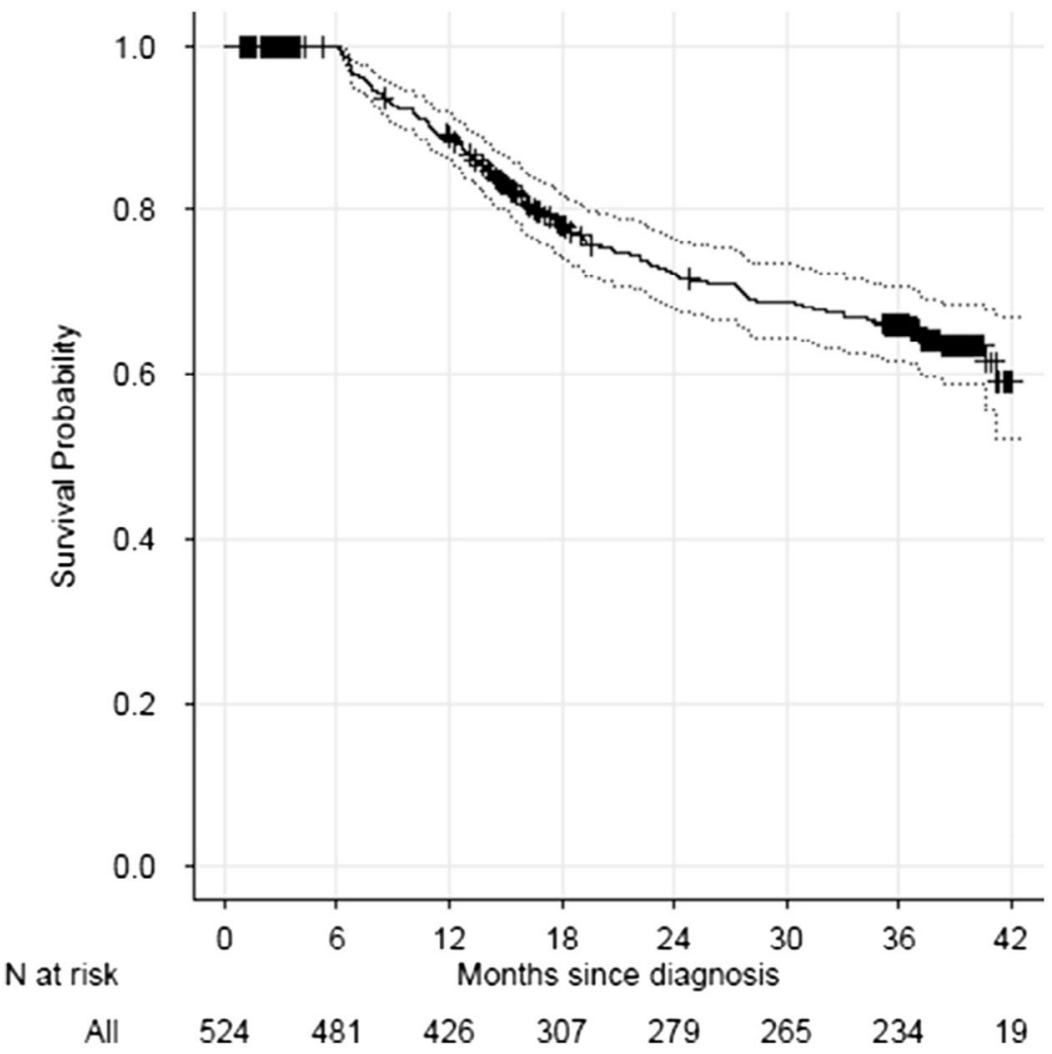

Figure 1.

Kaplan-Meier survival curve describing the probability of staying on active surveillance over 3 years. Censored values $(+)$ represent the last known follow-up of patients on active surveillance (AS). 


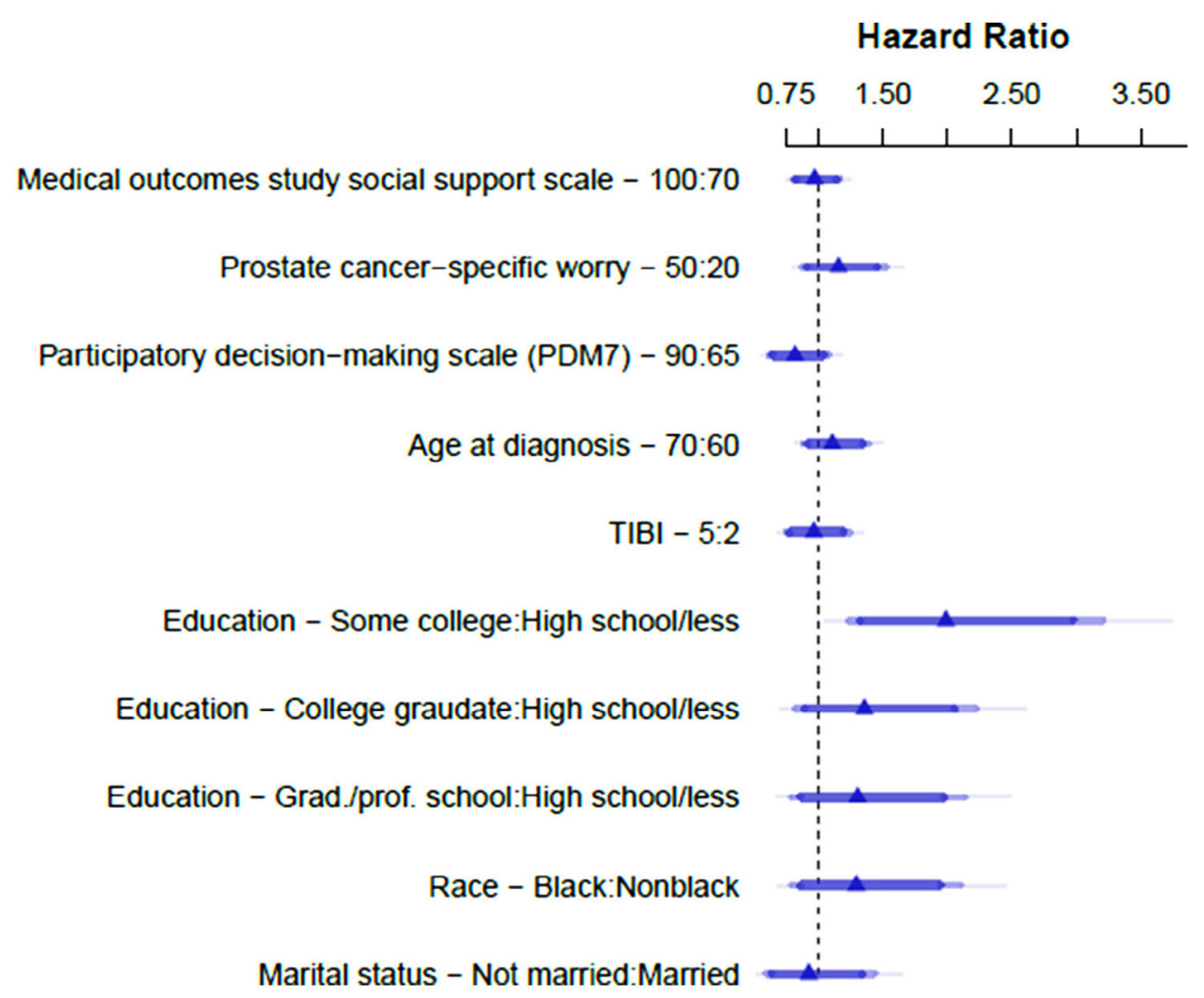

Figure 2.

Forest plot of multivariable hazard ratios. Triangles represent the predicted HRs of each factor in relation to time to discontinuing active surveillance. Triangles are flanked by $95 \%$ confidence interval $(\mathrm{CI})$ bands. Bands crossing the dotted line $(\mathrm{HR}=1)$ represent factors that did not significantly influence discontinuation of AS. (Color version available online.) 


\section{Table 1}

Patient-reported reasons for discontinuing active surveillance $(\mathrm{N}=77)^{*}$

\begin{tabular}{lc}
\hline Category of reason & \\
Medical & $53(69 \%)$ \\
Personal & $6(8 \%)$ \\
Both & $18(23 \%)$ \\
Medical reasons given & \\
Doctor said cancer worsened & $35(45.5 \%)$ \\
Higher Gleason score & $36(46.8 \%)$ \\
Change on digital rectal exam & $4(5.2 \%)$ \\
Increased volume on repeat biopsy & $23(29.9 \%)$ \\
Increased PSA & $47(61.0 \%)$ \\
Personal reasons given & \\
Spouse encouragement & $12(15.6 \%)$ \\
"I just decided" & $19(24.7 \%)$ \\
\hline
\end{tabular}

* Sections may not add up to $100 \%$ owing to multiple responses from individual patients. 